(2) Open Access Full Text Article

\title{
Mortality Rate of Trauma Patients with ESI Triage Level I-2 Who Underwent Computerized Tomography-PANSCAN versus Conventional Computerized Tomography Scan
}

\section{Sorravit Savatmongkorngul (iD \\ Chaiyaporn Yuksen (iD \\ Wapee Maspol \\ Pungkava Sricharoen (iD \\ Sirote Wongwaisayawan (D) \\ Chetsadakon Jenpanitpong (iD) \\ Sorawich Watcharakitpaisan (iD) \\ Parama Kaninworapan (iD \\ Konwachira Maijan (iD)}

Department of Emergency, Faculty of Medicine, Ramathibodi Hospital, Mahidol University, Bangkok, Thailand
Correspondence: Chaiyaporn Yuksen Email chaipool0634@hotmail.com
Objective: The treatment of severe trauma patients requires a fast and accurate method to diagnose life-threatening conditions. Computerized tomography (CT)-PANSCAN has been widely used for the last 20 years to diagnose many patients in critical condition. However, no research has been performed into the efficacy of CT-PANSCAN. This research aims to compare the mortality rate of trauma patients who underwent CT-PANSCAN versus conventional CT scan.

Methods: This retrospective cohort study enrolled patients who were at triage ESI level 1-2 in the emergency department of Ramathibodi Hospital from January 2013 to December 2018 and analyzed the mortality rate between those who underwent CT-PANSCAN and conventional CT scan.

Results: The study enrolled 123 trauma patients; 61 patients underwent CT-PANSCAN, whereas 62 patients underwent conventional CT scan. There were 1 and 7 patients who expired in the CT-PANSCAN and conventional CT scan groups, respectively. After multivariate regression analysis, the result revealed that patients who underwent CT-PANSCAN had a lower mortality rate (adjusted odds ratio $=0.023$; $\mathrm{p}$-value $=0.018$; 95\% CI $0.001-0.518$ ).

Conclusion: Undergoing a CT-PANSCAN can reduce the mortality rate in trauma patients, especially in ESI level 1, 2 traumatic patients, and CT-PANSCAN available facilities.

Keywords: whole body CT, trauma, mortality rate, death

\section{Introduction}

In 2019, the Global Burden of Disease Study reported that injuries (both intentional and unintentional) were the leading cause of death worldwide. Deaths occurred in 4.3 million population, which represents $7.6 \%$ of all causes of mortality. ${ }^{1}$ Severe traumatic injured patients usually present with life-threatening conditions caused by multiple organ injuries that require advanced trauma management. ${ }^{2}$ Early initiation of care for traumatically injured patients is important as it is associated with better outcomes. ${ }^{3}$

According to the Advanced Trauma Life Support (ATLS ${ }^{\circledR}$ ) protocol, to identify any life-threatening conditions, primary survey and its adjuncts (ie, vital signs and ECG monitoring, urinary and gastric catheterization, as well as diagnostic imaging) must be rapidly performed. ${ }^{4}$ In patients with neurological symptoms, the extent of physical examination is limited, and results should be cautiously interpreted, eg, signs indicated for cervical spine injury or peritoneal irritation may be obscured by those altered mental statuses or intoxicated. ${ }^{5}$ 
Diagnostic imaging including plain radiographs (Xrays), focused assessment with sonography for trauma (FAST), and computed tomography (CT) has become fundamental investigation tools for traumatically injured patients. ${ }^{4,6}$ However, there are some limitations to these modalities. For example, chest radiography can misdiagnose pneumothorax, ultrasonography is an operator-dependent procedure, and visualization may be limited in some organ regions. ${ }^{7}$ In traumatic brain injury, portable nearinfrared spectroscopy may be used for intracranial hemorrhage diagnosis but cannot be ruled out. ${ }^{8}$ In hemodynamically unstable patients, FAST positive is a high positive predictive value, and other modalities should always confirm a FAST-negative. ${ }^{9}$ Thus, organ-specific CT has been increasingly used as a radiological gold standard in recent years.

Many advantages support CT utilization in initial trauma evaluation. $\mathrm{CT}$ has more sensitivity and specificity to diagnosed life-threatening conditions than X-rays and FAST. The development of modern CT shortens the scanning time and results in high resolution. Its application can guide clinicians to decide which appropriate treatment should be provided for trauma patients. ${ }^{10-13}$

In some trauma centers, whole-body CT (WBCT) also known as CT PANSCAN - protocols have been implemented in trauma patients, especially those with multiple organ injuries. ${ }^{14}$

This yields superior advantages to conventional CT due to its accuracy in detecting unidentified injury and shorter investigation time. ${ }^{15-18}$ Several observational studies reported the improvement of clinical outcomes by decreasing mortality rate and hospital length of stay among trauma patients who underwent CT PANSCAN. ${ }^{19-22}$

However, the REACT-2 Study, ${ }^{23}$ an international, multicenter, randomized controlled trial, debated no statistical difference in the mortality rate for those who received CT PANSCAN or conventional scanning. In addition, this diagnostic procedure also extends the time spent in the emergency department (ED), especially in settings in which the CT room is not located within or near the ED. ${ }^{24}$ Prolonged diagnosis of definite intervention interval is associated with detrimental outcomes in trauma patients. ${ }^{25}$

Radiation exposure from diagnostic imaging is a safety issue that must be a concern. The average effective dose of whole-body scanning in adults is approximately $32 \mathrm{mSv}$, higher than the average range of other $\mathrm{CT}$ examinations (about $2-20 \mathrm{mSv}){ }^{26}$ Radiative exposure of more than 20 $\mathrm{mSv}$ increases the risk of cancer. This is based on the theory that doses above $20 \mathrm{mSv}$ have a cancer risk more significant than one in a thousand. ${ }^{27}$ There was a previous study that found that the implementation of CT PANSCAN protocol increased the odds of radiation exposure $>20 \mathrm{mSv}$ in trauma patients.

Recently, CT PANSCAN imaging has not been widely used in Thailand, and it is mostly available in tertiary-level or university-affiliated hospitals. Therefore, we hypothesized that CT PANSCAN imaging was associated with better outcomes in traumatically injured patients. Thus, the study aims to determine the association of performing a CT PANSCAN to the mortality of traumatically injured patients.

\section{Method}

\section{Study Setting and Participants}

The study was a retrospective cohort study conducted at the Emergency Department (ED) of Ramathibodi Hospital - a university-affiliated, level 1 trauma facilities in an urban area of Bangkok, Thailand. The Institutional Review Board (IRB) approved this study on Human Rights Related to Research Involving Human Subjects by Faculty of Medicine Ramathibodi Hospital, Mahidol University (Ethics code: MURA2018/453). The ethics committee did not require consent for this research because reviewing the medical record is the reason for the waiver and a statement covering patient data confidentiality and compliance with the Declaration of Helsinki.

All study subjects were trauma patients who were brought to the ED of Ramathibodi Hospital between January 1st, 2013 and December 31st, 2018. Inclusion criteria were patients those met the Emergency Severity Index (ESI) ${ }^{28}$ level 1 or 2 criteria. Exclusion criteria were patients aged less than 15 years and those who presented with traumatic cardiac arrest. Patients who were lost to follow-up or missing in outcome measurement were also excluded.

\section{Data Collection and Outcomes}

Data were retrieved from Ramathibodi Electronic Medical Record (EMR) database by using trauma record form. Study variables were collected include gender, age, underlying conditions, initial triage level based on emergency severity index (ESI), mechanism of injury, initial vital signs (respiratory rate, pulse rate, and systolic blood 
pressure, oxygen saturation), initial Glasgow coma scale, point-of-care testing hemoglobin level and drug or alcohol used status.

The primary outcomes were ED and 28-days mortality compared between patients who underwent CT PANSCAN and conventional CT.

\section{Sample Size Estimation}

We collected the data from the trauma patients for the pilot data. There were 34 and 30 patients who underwent CT PANSCAN and conventional CT, respectively. Among those who underwent CT PANSCAN and conventional CT, 1 (2.94\%) and 5 (16.67) patients expired, respectively.

According to the pilot study, P (death|CT PANSCAN) is $0.0294, \mathrm{P}$ (death $\mid \mathrm{CT}$ conventional) is 0.1667 , alpha is 0.05 , and beta is 0.8 . The sample size can be calculated as CT PANSCAN 73 and CT conventional 73. The total participants were 146 trauma patients.

\section{Statistical Analyses}

Data were analyzed using STATA version 14.0 (StataCorp, College Station, TX, USA). All study variables were compared between patients who underwent CT PANSCAN and conventional CT. The categorical data report as frequency and percentage. The numerical data report as mean and standard deviation or median and interquartile range. Exact probability test was used to compare categorical data. Independent $t$-test or Mann-Whitney $U$-test was used to compare numerical data, as appropriate.

The association between CT PANSCAN and mortality was calculated by univariable logistic regression, reports as unadjusted odds ratio and $95 \%$ confidence interval (CIs). Then, multivariable logistic regression was used to control the influence of confounding variables and reported as adjusted odds ratio, consistent with 95\% CI. All statistical analyses with p-value of less than 0.05 were considered statistically significant.

\section{Multivariable Analysis}

Age, gender, underlying conditions, mechanism of injury, respiratory rate, pulse rate, systolic $\mathrm{BP}$, oxygen saturation, Glasgow coma scale.

\section{Result}

Among the 65 patients who underwent CT-PANSCAN, 4 were excluded due to incomplete data. Thus, in total, there were 61 and 62 patients who underwent CT-PANSCAN and conventional CT scan, respectively.
The baseline characteristics of all patients are shown in Table 1. Of the 123 patients, 93 were males (75.60\%). Among the male patients, 47 (77.05\%) and 46 (74.19\%) underwent CT-PANSCAN and conventional CT scan, respectively. The average age of both groups is 42.66 years old (44.08 years in CT-PANSCAN and 41.35 years in conventional CT scans). The underlying diseases in each group were similar.

Although the patients' vital signs, respiratory rates, pulse rates, and blood pressures had similar means and standard deviations, there was a difference in the Glasgow Coma Scale (GCS) and oxygen saturation. Those who underwent CT-PANSCAN and conventional CT scan had a mean GCS of 11.28 and 12.90 and a mean oxygen saturation of $92.80 \%$ and $96.70 \%$, respectively.

For the Injury Severity Score (ISS) 2, patients who underwent CT-PANSCAN and conventional CT scan had a medium score of 29 and 19.5, respectively. There were also differences in the ESI triage-level ratio, as shown in Table 1.

For the patient outcomes, the mortality rate of patients for both modalities was 5.69\% (7 patients), 1 patient (1.64\%) who underwent CT-PANSCAN and 6 patients $(9.68 \%)$ who underwent a conventional scan, as shown in Table 1.

The 28-day number of mortalities was 18 (14.63\%), 11 $(18.03 \%)$, and 7 (11.29) patients for CT-PANSCAN and conventional CT scan, respectively, as shown in Table 1.

The number of mortalities in both groups was analyzed and compared using multivariate logistic regression using STATA $^{\circledR}$ version 14.2. The results had shown that the Trauma patients with ESI triage levels 1 and 2 who underwent CT-PANSCAN had a significantly lower mortality rate than those who underwent conventional $\mathrm{CT}$ scan (adjusted odds ratios $=0.023 ; \mathrm{p}$-value $=0.018 ; 95 \% \mathrm{CI}$ 0.001-0.518), as shown in Table 2.

Undergoing a CT-PANSCAN can reduce the mortality rate in trauma patients, promptly provides a diagnosis of life-threatening situations, results in better decision-making for treatments, is more accurate and more precise and preferable to ultrasound, which is operator-dependent.

\section{Discussion}

The results showed that patients in both the CTPANSCAN and conventional CT scan groups had similar baseline characteristics, but the differences in the mean GCS scores and oxygen saturation in the patients of both groups were interpreted as those that underwent CT- 
Table I Baseline Characteristics of Patients Who Underwent CT-PANSCAN versus Conventional CT

\begin{tabular}{|c|c|c|c|}
\hline & CT-PANSCAN (N = 61) & Conventional CT $(\mathrm{N}=62)$ & p-value \\
\hline Gender male - n (\%) & 47 (77.05) & $46(74.19)$ & 0.834 \\
\hline Age - years & $44.08 \pm 20.53$ & $41.35 \pm 20.54$ & 0.463 \\
\hline Body weight - Kg & $63.76 \pm 11.62$ & $61.32 \pm 11.94$ & 0.254 \\
\hline Height - cm & $165.70 \pm 9.76$ & $164.50 \pm 10.70$ & 0.516 \\
\hline $\mathrm{BMI}-\mathrm{Kg} / \mathrm{m}^{2}$ & $23.13 \pm 3.14$ & $22.62 \pm 4.23$ & 0.495 \\
\hline $\begin{array}{l}\text { Underlying conditions - n (\%) } \\
\text {-Hypertension } \\
\text {-Diabetes mellitus } \\
\text {-Dyslipidemia } \\
\text {-Ischemic heart disease } \\
\text {-Cerebrovascular disease } \\
\text {-Bleeding disorder } \\
\text {-Others }\end{array}$ & $\begin{array}{c}8(13.1 \mathrm{I}) \\
4(6.56) \\
8(13.1 \mathrm{I}) \\
3(4.92) \\
2(3.28) \\
3(4.92) \\
5(8.20)\end{array}$ & $\begin{array}{c}6(9.68) \\
4(6.45) \\
6(9.68) \\
2(3.23) \\
1(1.61) \\
5(8.06) \\
10(16.13)\end{array}$ & $\begin{array}{l}0.583 \\
1.000 \\
0.583 \\
0.680 \\
0.619 \\
0.717 \\
0.270\end{array}$ \\
\hline $\begin{array}{l}\text { Mechanism of injury - n (\%) } \\
\text {-Blunt } \\
\text {-Penetrating } \\
\text {-Blast }\end{array}$ & $\begin{aligned} 57 & (93.44) \\
2 & (3.28) \\
2 & (3.28)\end{aligned}$ & $\begin{array}{c}57(91.94) \\
5(8.06) \\
-\end{array}$ & 0.276 \\
\hline Drug/alcohol intoxication - $\mathrm{n}(\%)$ & $4(6.56)$ & $8(12.90)$ & 0.363 \\
\hline $\begin{array}{l}\text { ESI triage level - n (\%) } \\
\text {-ESI level I } \\
\text {-ESI level } 2\end{array}$ & $\begin{array}{l}29(48.33) \\
31(51.67)\end{array}$ & $\begin{array}{l}\text { II (I7.74) } \\
5 \mathrm{I}(82.26)\end{array}$ & $<0.001$ \\
\hline $\begin{array}{l}\text { Initial vital signs } \\
\text {-Respiratory rate }(\mathrm{bpm}) \\
\text {-Pulse rate }(\mathrm{bpm}) \\
\text {-Systolic BP }(\mathrm{mmHg})\end{array}$ & $\begin{array}{c}22.56 \pm 6.83 \\
94.70 \pm 30.90 \\
115.05 \pm 53.90\end{array}$ & $\begin{array}{c}22.29 \pm 3.73 \\
98.87 \pm 25.96 \\
|4| .84 \pm 34.1 \mid\end{array}$ & $\begin{array}{l}0.788 \\
0.419 \\
0.001\end{array}$ \\
\hline Oxygen saturation - \% & $92.80 \pm 18.41$ & $96.69 \pm 2.24$ & 0.101 \\
\hline $\begin{array}{l}\text { Glasgow coma scale - n (\%) } \\
\text {-Average } \\
-13-15 \\
-9-12 \\
-3-8\end{array}$ & $\begin{array}{l}11.28 \pm 4.47 \\
33(54.10) \\
10(16.39) \\
18(29.51)\end{array}$ & $\begin{array}{l}12.90 \pm 3.63 \\
46(74.19) \\
6(9.68) \\
10(16.13)\end{array}$ & $\begin{array}{l}0.029 \\
0.060\end{array}$ \\
\hline Point-of-care hemoglobin - g/dL & $12.30 \pm 2.36$ & $13.18 \pm 1.47$ & 0.015 \\
\hline Injury severity score (ISS) & $29(18,48)$ & $19.5(7,29)$ & $<0.00 I^{*}$ \\
\hline $\begin{array}{l}\text { Medium (IQR) } \\
\text { Hospital length of stay - days }\end{array}$ & $10(2-19)$ & $2(I-7)$ & $<0.001 *$ \\
\hline $\begin{array}{l}\text { ED dispositions - n (\%) } \\
\text {-Trauma/surgical ward } \\
\text {-Intensive care unit } \\
\text {-Operating theatre } \\
\text {-Deceased }\end{array}$ & $\begin{array}{l}\text { II }(18.03) \\
44(72.13) \\
\text { I }(1.64) \\
\text { I }(1.64)\end{array}$ & $\begin{array}{c}30(48.39) \\
20(32.26) \\
- \\
6(9.68)\end{array}$ & $\begin{array}{c}<0.001 \\
<0.001 \\
0.496 \\
0.114\end{array}$ \\
\hline 28 days mortality - n (\%) & II (I8.03) & 7 (II.29) & 0.319 \\
\hline
\end{tabular}


Table 2 Objectives and Outcomes

\begin{tabular}{|l|l|c|c|c|}
\hline Factors & Crude Odds Ratio & Adjusted Odds Ratio & Adjusted p-value & $\mathbf{9 5 \%}$ Cl \\
\hline CT-PANSCAN & 0.156 & 0.023 & 0.018 & $0.001-0.518$ \\
ISS & 1.053 & 1.115 & 0.005 & $1.033-1.203$ \\
Age & 1.086 & 1.044 & 0.024 & $1.005-1.084$ \\
Hb & 1.594 & 1.029 & 0.893 & $0.679-1.558$ \\
\hline
\end{tabular}

PANSCAN were in a worse state than those who underwent conventional CT scan.

Although patients who underwent CT-PANSCAN were in a worse state, the multivariate logistic regression analysis showed that the mortality rate of patients who underwent CTPANSCAN was significantly lower (adjusted odds ratio = 0.023 ; $\mathrm{p}$-value $=0.018 ; 95 \%$ CI $0.001-0.518$ ).

According to Long et al ${ }^{15}$ CT-PANSCAN can decrease mortality, but this benefit was limited to only some groups. Moreover, Çorbacioğlu and Aksel ${ }^{10}$ showed that undergoing CT-PANSCAN can reduce the mortality rate of severe trauma patients. Study of Jiang et al is a metaanalysis comparison of whole-body $\mathrm{CT}$ and selective radiological imaging. Whole-body CT significantly reduces the mortality rate. ${ }^{3}$

The REACT-2 study, ${ }^{23}$ including all traumatic patients aged 18 years with unstable vital signs and life-threatening or severe injuries, shows that PAN-SCAN does not reduce in-hospital mortality. Our study enroll the ESI level 1 traumatic patient (life-threatening injuries or severe injury) and ESI level 2 traumatic patient (high-risk injuries patient) to candidates to CT-PANSCAN. In ESI level 2, CT-PANSCAM should be done in every patient, ESI level 1 should consider a patient's condition, operation room, and CT-PANSCAN available. In Ramathibodi Hospital, the CT-PANSCAN was always available 24 hours and located within the resuscitation room. There was no delay time for CT-PANSACN after primary resuscitation.

Along with previous reports, this research has shown that undergoing a CT-PANSCAN can reduce the mortality rate in trauma patients, promptly diagnose life-threatening situations, result in better decision-making for treatments, and be more accurate and preferable than ultrasound, which is operator dependent.

The overall cost of CT-PANSCAN in Ramathibodi Hospital was 600 US per case. Therefore, CT-PANSCAN in every traumatic patient has a significant financial impact. $^{24}$ The study by Oosthuizen et al recommended using CT-PANSCAN to rule out significant injuries.
Clinically negative scans are valuable in obtunded patients, intubated, or have major distracting injuries. Clinical assessment and alternative imaging modalities may suffice in traumatic patients with full consciousness, no intubation, and significant distracting injuries. ${ }^{29}$ Consequently, we recommend selecting the traumatic patient in ESI levels 1 and 2 to candidate to CTPANSCAN.

CT-PANSCAN should not delay the definite operation in critically injured patients. In hemodynamic unstable traumatic patients, the delay time for CT-PANSCAN may result in a poor outcome. Therefore, clinicians should be aware and select the appropriate patient for CTPANSCAN. ${ }^{30}$

\section{Limitations}

First, this was a retrospective study. Some factors could not be controlled, so statistical methods were required to analyze the results. Second, selection is biased by the retrospective design. Third, CT-PANSCAN is not widely available in our country. This study was conducted at a university hospital with a tertiary trauma center, the first public health study in Thailand. To validate our results, further studies should include more patients who have undergone CT-PANSCAN.

\section{Conclusion}

Undergoing a CT-PANSCAN can reduce the mortality rate in trauma patients, especially in ESI level 1,2 traumatic patients, and CT-PANSCAN available facilities.

\section{Disclosure}

The authors report no conflicts of interest in this work.

\section{References}

1. GBD 2019 Diseases and Injuries Collaborators. Global burden of 369 diseases and injuries in 204 countries and territories, 1990-2019: a systematic analysis for the Global Burden of Disease Study 2019 [published correction appears in Lancet]. Lancet. 2020;396 (10258):1204-1222. 
2. Gunning A, van Heijl M, van Wessem K, Leenen L. The association of patient and trauma characteristics with the health-related quality of life in a Dutch trauma population. Scand J Trauma Resusc Emerg Med. 2017;25(1):41. doi:10.1186/s13049-017-0375-z

3. Jiang L, Ma Y, Jiang S, et al. Comparison of whole-body computed tomography vs selective radiological imaging on outcomes in major trauma patients: a meta-analysis. Scand J Trauma Resusc Emerg Med. 2014;22(1):54. doi:10.1186/s13049-014-0054-2

4. Committee on Trauma, American College of Surgeons. Advanced Trauma Life Support Student Course Manual. 10th ed. Chicago, IL: American College of Surgeons; 2018.

5. Mayumi T, Yoshida M, Tazuma S, et al. The practice guidelines for primary care of acute abdomen 2015. Jpn J Radiol. 2016;34(1):80115. doi:10.1007/s11604-015-0489-z

6. Healy DA, Hegarty A, Feeley I, Clarke-Moloney M, Grace PA, Walsh SR. Systematic review and meta-analysis of routine total body CT compared with selective CT in trauma patients. Emerg Med J. 2014;31:101-108. doi:10.1136/emermed-2012-201892

7. Hoffstetter P, Dornia C, Schäfer S, et al. Diagnostic significance of rib series in minor thorax trauma compared to plain chest film and computed tomography. J Trauma Manag Outcomes. 2014;8:10. doi:10.1186/1752-2897-8-10

8. Yuksen C, Sricharoen P, Puengsamran N, Saksobhavivat N, Sittichanbuncha Y, Sawanyawisuth K. Diagnostic properties of a portable near-infrared spectroscopy to detect intracranial hematoma in traumatic brain injury patients. Eur J Radiol Open. 2020;7:100246. doi:10.1016/j.ejro.2020.100246

9. Engles S, Saini NS, Rathore S. Emergency focused assessment with sonography in blunt trauma abdomen. Int J Appl Basic Med Res. 2019;9:193-196. doi:10.4103/ijabmr.IJABMR_273_19

10. Self ML, Blake AM, Whitley M, Nadalo L, Dunn E. The benefit of routine thoracic, abdominal, and pelvic computed tomography to evaluate trauma patients with closed head injuries. Am J Surg. 2003;186:609-614. doi:10.1016/j.amjsurg.2003.08.003

11. van Vugt R, Kool DR, Deunk J, et al. Effects on mortality, treatment, and time management as a result of routine use of total body computed tomography in blunt high-energy trauma patients. J Trauma Acute Care Surg. 2012;72(3):553-559. doi:10.1097/ TA.0b013e31822dd93b

12. Sampson MA, Colquhoun KB, Hennessy NL. Computed tomography whole body imaging in multi-trauma: 7 years experience. Clin Radiol. 2006;61(4):365-369. doi:10.1016/j.crad.2005.12.009

13. Okamoto K, Norio H, Kaneko N, Sakamoto T, Kaji T, Okada Y. Use of early-phase dynamic spiral computed tomography for the primary screening of multiple trauma. Am J Emerg Med. 2002;20(6):528-534. doi:10.1053/ajem.2002.34802

14. Treskes K, Saltzherr TP, Luitse JS, Beenen LF, Goslings JC. Indications for total-body computed tomography in blunt trauma patients: a systematic review. Eur J Trauma Emerg Surg. 2017;43 (1):35-42. doi:10.1007/s00068-016-0711-4

15. Long B, April MD, Summers S, Koyfman A. Whole body CT versus selective radiological imaging strategy in trauma: an evidence-based clinical review. Am J Emerg Med. 2017;35(9):1356-1362. doi:10.1016/j.ajem.2017.03.048

16. Caputo ND, Stahmer C, Lim G, Shah K. Whole-body computed tomographic scanning leads to better survival as opposed to selective scanning in trauma patients: a systematic review and meta-analysis. $J$ Trauma Acute Care Surg. 2014;77(4):534-539. doi:10.1097/ TA.0000000000000414
17. Wurmb TE, Frühwald P, Hopfner W, et al. Whole-body multislice computed tomography as the first line diagnostic tool in patients with multiple injuries: the focus on time. $J$ Trauma. 2009;66:658-665.

18. Wurmb TE, Quaisser C, Balling H, et al. Whole-body multislice computed tomography (MSCT) improves trauma care in patients requiring surgery after multiple trauma. Emerg Med J. 2011;28 (4):300-304. doi:10.1136/emj.2009.082164

19. Huber-Wagner S, Biberthaler P, Haberle S, et al. Whole-body CT in haemodynamically unstable severely injured patients - a retrospective, Multicentre Study. PLoS One. 2013;8(7):e68880. doi:10.1371/ journal.pone. 0068880

20. Sabzghabaei A, Shojaee M, Kariman H, Manouchehrifar M, Heydari K, Sohrabi S. Pan vs. selective computed tomography scans in management of multiple trauma patients; a brief report. Emerg. 2017;5(1):e38.

21. Salim A, Sangthong B, Martin M, Brown C, Plurad D, Demetriades D. Whole body imaging in blunt multisystem trauma patients without obvious signs of injury: results of a prospective study. Arch Surg. 2006;141:468-473. doi:10.1001/archsurg.141.5.468

22. Yeguiayan JM, Yap A, Freysz M, et al; FIRST Study Group. Impact of whole-body computed tomography on mortality and surgical management of severe blunt trauma. Crit Care. 2012;16:R101. doi:10.1186/cc11375

23. Sierink JC, Treskes K, Edwards MJ, et al; REACT-2 study group. Immediate total-body CT scanning versus conventional imaging and selective CT scanning in patients with severe trauma (REACT-2): a randomised controlled trial. Lancet. 2016;388(10045):673-683. doi:10.1016/S0140-6736(16)30932-1

24. Bashir AA, Kong VY, Weale RD, et al. Quantifying the burden of trauma imaging on the CT scan service at a major trauma centre in South Africa. S Afr J Surg. 2019;57:48-53. doi:10.17159/2078-5151/ 2019/v57n2a2836

25. Murao S, Yamakawa K, Kabata D, et al. Effect of earlier door-to-CT and door-to-bleeding control in severe blunt trauma: a Retrospective Cohort Study. J Clin Med. 2021;10:1522. doi:10.3390/jcm10071522

26. Mettler FA Jr, Huda W, Yoshizumi TT, Mahesh M. Effective doses in radiology and diagnostic nuclear medicine: a catalog. Radiology. 2008;248(1):254-263. doi:10.1148/radiol.2481071451

27. Radiation Protection Series Publication No. 8. Exposure of Humans to Ionizing Radiation for Research Purposes. Australian Radiation Protection and Nuclear Safety Agency; 2005:13-14.

28. Gilboy N, Tanabe P, Travers DA, Rosenau AM, Eitel DR. Emergency Severity Index, Version 4: Implementation Handbook. AHRQ Publication No. 05-0046-2. Rockville, MD: Agency for Healthcare Research and Quality; May 2005.

29. Oosthuizen GV, Bruce JL, Bekker W, Shangase N, Laing GL, Clarke DL. Pan computed tomography for blunt polytrauma: are we doing too many? $S$ Afr Med J. 2016;106(8):801-803. doi:10.7196/ SAMJ.2016.v106i8.10376

30. Neal MD, Peitzman AB, Forsythe RM, et al. Over reliance on computed tomography imaging in patients with severe abdominal injury: is the delay worth the risk? J Trauma. 2011;70:278-284. 
Open Access Emergency Medicine

Dovepress

\section{Publish your work in this journal}

The Open Access Emergency Medicine is an international, peerreviewed, open access journal publishing original research, reports, editorials, reviews and commentaries on all aspects of emergency medicine. The manuscript management system is completely online

and includes a very quick and fair peer-review system, which is all easy to use. Visit http://www.dovepress.com/testimonials.php to read real quotes from published authors.

Submit your manuscript here: https://www.dovepress.com/open-access-emergency-medicine-journal 